\title{
Predictors of Low Uptake of Prenatal Tetanus Toxoid, Reduced Diphtheria Toxoid, and Acellular Pertussis Immunization in Privately Insured Women in the United States
}

\author{
Anne M. Butler, $\mathrm{PhD}^{1,{ }^{*}}$, J. Bradley Layton, $\mathrm{PhD}^{1}$, Dongmei Li, MS ${ }^{1}$, Michael G. Hudgens, \\ $\mathrm{PhD}^{2}$, Kim A. Boggess, MD ${ }^{3}$, Leah J. McGrath, PhD $^{4}$, David J. Weber, MD, MPH ${ }^{1,5}$, and \\ Sylvia Becker-Dreps, MD, MPH 6 \\ 1'Department of Epidemiology, University of North Carolina at Chapel Hill, Chapel Hill, NC \\ ${ }^{2}$ Department of Biostatistics, University of North Carolina at Chapel Hill, Chapel Hill, NC \\ ${ }^{3}$ Department of Obstetrics \& Gynecology, University of North Carolina at Chapel Hill, Chapel Hill, \\ NC \\ ${ }^{4} \mathrm{RTI}$ Health Solutions, Research Triangle Park, NC \\ ${ }^{5}$ Division of Infectious Diseases, University of North Carolina at Chapel Hill, Chapel Hill, NC \\ ${ }^{6}$ Department of Family Medicine, University of North Carolina at Chapel Hill, Chapel Hill, NC
}

\section{Abstract}

OBJECTIVE-To examine the uptake of prenatal tetanus toxoid, reduced diphtheria toxoid, and acellular pertussis (Tdap) immunization among pregnant women in the United States.

\begin{abstract}
METHODS-Using MarketScan data, we conducted a historical cohort study among pregnant women with employer-based commercial insurance in the United States who delivered between January 1, 2010 and December 31, 2014. We examined temporal trends of uptake, predictors of uptake, and timing of Tdap immunization.
\end{abstract}

\begin{abstract}
RESULTS-Among 1,222,384 eligible pregnancies in 1,147,711 unique women, receipt of prenatal Tdap immunization increased from $0.0 \%$ of women who delivered in January 2010 to 9.8\% who delivered in October 2012 (the date of the recommendation by the Advisory Committee on Immunization Practices for Tdap during every pregnancy) to $44.4 \%$ who delivered in December 2014. Among women who received Tdap during pregnancy, the majority were immunized between 27 weeks and 36 weeks 6 days of gestation, per the ACIP recommendation. In multivariable analyses among women who delivered between November 2012 and December 2014, rates of prenatal Tdap immunization were lower for women under 25 years of age (e.g.,
\end{abstract}

\footnotetext{
"CORRESPONDING AUTHOR'S CONTACT INFORMATION UNC Gillings School of Global Public Health, University of North Carolina at Chapel Hill, McGavran-Greenberg, CB \# 7435, Chapel Hill, NC 27599-7435, Phone: (314) 952-4451, Fax: (919) 966.2089, anne.butler@unc.edu.

Financial Disclosure

Sylvia Becker-Dreps has received investigator-initiated research funds from Pfizer for an unrelated study on pneumococcal vaccines. David J. Weber served on Speaker's bureaus and as a vaccine consultant (Merck, Pfizer). The other authors did not report any potential conflicts of interest.

Each author has indicated that he/she has met the journal's requirements for authorship.
} 
20-24 vs. 30-34 years rate ratio [RR], 0.83, 95\% confidence interval [CI], 0.85-0.88), with other children (e.g., 3 vs. 0 children: RR, 0.86, 95\% CI, 0.84-0.88), residing in the South vs. Midwest (RR, 0.81, 95\% CI, 0.80-0.82), or with emergency department visits in early pregnancy (RR, 0.93, 95\% CI, 0.92-0.95). The proportion of pregnant women who received prenatal Tdap increased with increasing gestational age at birth.

CONCLUSION-By the end of 2014, less than half of pregnant women in the US were receiving prenatal Tdap immunization. Implementation and dissemination strategies are needed to increase Tdap coverage among pregnant women, especially those who are young, have other children, or reside in the South.

\section{Graphical abstract}

PRÉCIS: Less than half of pregnant women are receiving guideline-recommended tetanus toxoid, reduced diphtheria toxoid, and acellular pertussis (Tdap) immunization.

\section{INTRODUCTION}

Pertussis incidence has been rising in the United States (US) during the past decade, ${ }^{1}$ with a 2014 rate of 169 pertussis cases per 100,000 infants under 6 months of age. ${ }^{1}$ Disease in infants is associated with hospitalization, apnea, pneumonia, and death. ${ }^{1-3}$ The Centers for Disease Control and Prevention (CDC) recommends that infants receive the primary series of diphtheria and tetanus toxoids and acellular pertussis vaccine (DTaP) at 2, 4, and 6 months of age and 2 booster doses later in childhood to protect against pertussis infection. ${ }^{4}$ However, maximal protection is not attained until after the third dose at 6 months of age,, 6 leaving young infants at higher risk of pertussis. Therefore, the Advisory Committee on Immunization Practices (ACIP) has made several recent recommendations which were adopted by the CDC regarding prenatal or postpartum immunization of mothers with adult tetanus toxoid, reduced diphtheria toxoid, and acellular pertussis (Tdap) immunization. ${ }^{7-9}$

Little is known about national adherence to CDC guidelines for Tdap immunization during pregnancy. Several studies suggest low uptake, but results are dated and limited to select states or regions of the US. ${ }^{10-13}$ Knowledge about individual, provider, and ecological predictors of prenatal Tdap uptake is important to inform public health efforts to improve coverage, yet no large studies have identified factors associated with prenatal Tdap receipt. Although current evidence is somewhat conflicting, ${ }^{14-17}$ the timing of Tdap receipt during pregnancy may affect the quantity of maternal anti-pertussis antibodies transferred.

Therefore, we sought to examine temporal trends in uptake, predictors of uptake, and timing of Tdap immunization in a large national sample of pregnant women in the US.

\section{MATERIALS AND METHODS}

Using data from the MarketScan Commercial Claims and Encounters Databases (Copyright (C) 2014 Truven Health Analytics Inc. All Rights Reserved), we identified a historical cohort of women with evidence of a pregnancy outcome at $\geq 20$ weeks gestational age between January 1, 2010 and December 31, 2014. The MarketScan databases capture patient-level data on inpatient, outpatient, and prescription drug claims from approximately 100 large employers and health plans that insure employees and their dependents in all 50 states. In 
2010 , the database included approximately 920,000 pregnant women, corresponding to almost $25 \%$ of the birth cohort in the US and 50\% of the birth cohort in the US with commercial insurance. ${ }^{18,19}$ We required continuous enrollment in a payer plan between the estimated date of pregnancy onset and one week postpartum. We defined the delivery date as the billing date of an International Classification of Clinical Diseases, Ninth Revision, Clinical Modification (ICD-9-CM) code for outcome of delivery, V27.0-V27.6. The date of pregnancy onset was estimated by subtracting the estimated gestational age from the delivery date (see Appendix 1, available online at http://links.lww.com/xxx). Multiple pregnancies of the same woman during the study period were eligible for inclusion. To reduce outcome misclassification from vaccines administered without submitting billing claims to insurance, we excluded women $\leq 18$ years of age residing in 13 states with universal Vaccines for Children (VFC) programs (Alaska, Hawaii, Maine, Massachusetts, New Hampshire, New Mexico, North Carolina, South Dakota, Rhode Island, Vermont, Washington, Wisconsin, and Wyoming). ${ }^{20}$

We evaluated immunization receipt during a time period spanning several changing CDC recommendations regarding prenatal or postpartum immunization of women. In 2006, the ACIP recommended postpartum immunization of mothers with adult tetanus-diphtheriaacellular pertussis immunization (Tdap) for those who had not previously received Tdap, as part of a strategy known as "cocooning." "This strategy had limited success in reducing rates of pertussis. ${ }^{21,22}$ In response, in June 2011, the ACIP voted to recommend that all pregnant women receive a single Tdap booster in late pregnancy ( $\geq 20$ weeks gestation) if they had not previously received Tdap, in order to convey passive immunity to young infants through transplacental IgG antibody transfer. ${ }^{7}$ Following the recommendation, immunogenicity data showed that anti-pertussis antibodies are short-lived and Tdap immunization in a prior pregnancy may not protect infants in subsequent pregnancies. ${ }^{17}$ Therefore, in October 2012, ACIP recommended the administration of Tdap in each pregnancy, further defining optimal timing for administering Tdap as the time window between 27 and 36 weeks 6 days gestation $^{8}$; the recommendation was approved by the CDC in February 2013. ${ }^{8}$

We classified Tdap immunization status using inpatient and outpatient ICD-9 procedure codes 99.37, 99.39, V06.1 and Current Procedural Terminology (CPT) code 90715, as immunization services are coded and billed separately outside the global obstetrician packages employed by some insurers. ${ }^{23}$ We used the October 2012 ACIP recommendation to categorize optimal timing of Tdap immunization during pregnancy for all calendar years, 2010-2014. We categorized Tdap immunization as early prenatal ( $<27$ weeks gestation), optimally-timed prenatal ( 27 weeks to 36 weeks 6 days gestation), late prenatal ( 237 weeks gestation), postpartum ( $\leq$ week postpartum), and none (no evidence of prenatal or postpartum Tdap immunization). For women who received more than one Tdap immunization during pregnancy, categorization was based on the earliest recorded Tdap immunization.

Demographic characteristics included maternal age at pregnancy onset; delivery year; number of other dependent children; insurance type; network of provider type (in-network, out-of-network or mix of networks, no service); geographic region; state; residence within a metropolitan statistical area (defined by the US Census Bureau as a geographical region with 
a high population density at its core and close economic ties throughout the area). ${ }^{24}$ Healthcare utilization variables, which served as proxy metrics to identify complicated pregnancies, included emergency department visits prior to 20 weeks gestation; and overnight hospitalizations prior to 20 weeks gestation. Health insurance type was categorized as Preferred Provider Organization (PPO) or Exclusive Provider Organization (EPO); Health Maintenance Organization (HMO); Point of Service (POS) or POS with capitation; Consumer Directed Health Plan (CDHP); or other (i.e., basic, comprehensive, or high-deductible plans). Number of other dependent children was defined as the number of other children $\leq 18$ years of age covered by the same insurance plan within the household on the delivery date.

Descriptive statistics were generated for individual, provider, and ecological characteristics by receipt and timing of Tdap immunization during pregnancy. Analyses were further stratified by relevant individual, provider, and ecological characteristics.

To identify individual, provider, and ecological predictors of prenatal Tdap immunization, multivariable Cox proportional hazards regression models were used to estimate adjusted hazard ratios (HRs), interpreted as rate ratios (RRs), as well as corresponding 95\% confidence intervals (CIs). Individuals were censored at the earliest of the following; seven days after birth, end of continuous enrollment in a health plan, or end of study. The robust sandwich estimate of the covariance matrix was specified to allow for clustering at the individual-level. Potential predictors of Tdap immunization were determined a priori. Mothers with missing data on any potential predictors (6.4\%) were excluded from these analyses.

To understand the relationship between receipt of prenatal Tdap and gestational age at birth, proportions of pregnant women who received prenatal Tdap were calculated by categories of preterm, term, and post-term births in the time period after the second recommendation of Tdap in pregnancy (November 2012 to December 2014). Corresponding 95\% CIs were also calculated.

To examine geographic variation, prenatal Tdap coverage was calculated by state in the time period after the second ACIP recommendation of Tdap in pregnancy (November 2012 to December 2014). A map of the U.S. was created with state values categorized by the quintile distribution of the data.

In sensitivity analyses, analyses were stratified by immediate and later periods after the October 2012 ACIP recommendation (i.e., 11/2012-12/2013; and 1/2014-12/2014).

SAS software version 9.3 (SAS Institute, Cary, NC) and R Statistical Software version 3.3 were used to perform all analyses. The institutional review board at the University of North Carolina at Chapel Hill approved this research.

\section{RESULTS}

Of the 1,222,384 eligible pregnancies among 1,147,711 unique women identified in the database (Appendix 2, available online at http://links.lww.com/xxx), prenatal or postpartum 
Tdap was administered in 241,543 (19.8\%) pregnancies. Specifically, $2.4 \%$ of pregnancies received early prenatal Tdap, $11.0 \%$ of pregnancies received optimally-timed prenatal Tdap, $1.0 \%$ of pregnancies received late prenatal Tdap, and $5.4 \%$ of pregnancies received postpartum Tdap. (Table 1; Appendix 3, available online at http://links.lww.com/xxx) Compared to women who received prenatal Tdap, women who did not receive prenatal or postpartum Tdap were more likely to have other dependent children, to live in the South, to have an in-network provider, or to have had an overnight hospitalization during pregnancy.

The proportion of pregnant women who received prenatal Tdap increased with increasing gestational age at birth: $5.9 \%$ for extreme preterm ( $<28$ weeks), $11.3 \%$ for very preterm ( 28 to $<32$ weeks), $24.5 \%$ for moderate to late preterm (32 to $<37$ weeks), $28.4 \%$ for term, and $34.6 \%$ for post-term (Appendix 4, available online at http://links.lww.com/xxx).

The proportion of pregnant women who received prenatal Tdap increased from $0.0 \%$ of women who delivered in January 2010 to $1.6 \%$ of women who delivered in June 2011 (i.e., the date of first ACIP recommendation for Tdap during pregnancy) to $9.8 \%$ of women who delivered in October 2012 (i.e., date of second ACIP recommendation for Tdap during pregnancy); thereafter, prenatal Tdap immunization increased sharply to $44.4 \%$ of women who delivered in December 2014. The timing of Tdap administration changed over time (Figure 1). From October 2012 to December 2014, Tdap immunization increased slightly for the early ( $2.2 \%$ to $4.2 \%)$ and late prenatal period (1.3\% to $1.9 \%)$, increased sharply for the optimally-timed prenatal period (6.3\% to $38.3 \%$ ), and decreased slightly for the postpartum period $(7.1 \%$ to $4.1 \%)$.

Temporal trends in prenatal Tdap immunization differed across subgroups (Figure 2). Since October 2012, the uptake of Tdap immunization was consistently higher among women of older age or with fewer dependent children. Regionally, Tdap immunization was highest in the West from mid-2010 to early 2013; and thereafter, highest in the Midwest and lowest in the South (Figures 2 and 3).

Among women who delivered between November 2012 and December 2014, variation in rates of Tdap immunization were observed across subgroups (Appendix 5, available online at http://links.lww.com/xxx). Compared to women 30-34 years of age, rates of Tdap receipt were $17 \%$ (95\% CI, 15-20\%) lower among women $14-19$ years of age, $13 \%$ (95\% CI, $12-15 \%)$ lower among women 20-24 years of age, and 5\% (95\% CI, 4-6\%) lower among women 25-29 years of age. Tdap rates decreased with increasing number of other dependent children. Geographically, Tdap rates were higher among women residing within a metropolitan statistical area. Compared to women living in the Midwest, rates of Tdap receipt were 19\% (95\% CI, 18-20\%) lower in the South, 18\% (95\% CI, 17-19\%) lower in the West, and 16\% (95\% CI, 14-17\%) lower in the Northeast. Rates of Tdap receipt were lower among women who had an emergency department visit in early pregnancy. Tdap receipt varied by network of provider and health plan type. There were no appreciable differences in the predictors of Tdap receipt between immediate and later time periods after the October 2012 ACIP recommendation (data not shown). 


\section{DISCUSSION}

We conducted a national study of the uptake of prenatal Tdap immunization among 1.2 million privately-insured pregnant women in the US from 2010 to 2014. We observed a steep increase in prenatal Tdap immunization after the October 2012 ACIP recommendation for Tdap immunization during every pregnancy. Yet, national coverage remained below $45 \%$ by the end of 2014. The majority of prenatal Tdap recipients were immunized between 27 weeks and 36 weeks 6 days gestation, as encouraged by the ACIP recommendation. Receipt of postpartum Tdap was low and increasingly rare, despite the high proportion of unimmunized women at delivery, representing lost opportunities for prevention of infant pertussis.

For the period before the 2012 ACIP recommendation, our findings of low yet increasing coverage are consistent with published reports (i.e., Michigan data on Medicaid-enrollees and regional Vaccine Safety Datalink (VSD) data on insured patients seeking care at large integrated healthcare systems). ${ }^{11,12}$ For the period after the 2012 ACIP recommendation, our estimates of prenatal coverage are lower than other studies. One possible explanation for this discrepancy is that existing studies were conducted in states with exceptionally high 2012 pertussis incidence, which urged local efforts to increase prenatal coverage. A study in Wisconsin, the state with the highest 2012 pertussis incidence, reported an increase in prenatal Tdap coverage from January 2013 (13.8\%) to March 2014 (51.0\%). ${ }^{10,25}$ A VSD study reported $41.7 \%$ prenatal Tdap coverage in 2013 , which is likely attributable to immunization campaigns in response to exceptionally high 2012 pertussis incidence in several of these VSD states. ${ }^{13,25}$ Our most recent national estimate of prenatal Tdap coverage (44.4\% in December 2014) is slightly lower than national prenatal influenza coverage during the 2014/2015 influenza season (50.3\%), according to a CDC survey of 1702 women with a mix of private, public, or no insurance. ${ }^{26}$

Existing epidemiological studies to identify predictors of Tdap or influenza immunization during pregnancy are limited by small sample size, univariate methods, and limited followup after relevant ACIP recommendations. ${ }^{27,28}$ In multivariable analyses, we identified substantial variability in prenatal Tdap immunization across patient subgroups. Specifically, rates of prenatal Tdap immunization were lower among women who were younger, had more dependent children, experienced an emergency department visit during early pregnancy, or accessed an out-of-network provider. Prenatal Tdap immunization also varied regionally, which is consistent with national survey data on Tdap immunization among women regardless of pregnancy status. ${ }^{29,30}$ Specifically, prenatal Tdap receipt was highest in the Midwest and lowest in the South. Racial disparities in vaccine receipt may partially explain the geographic distribution, as Black women are less likely to receive prenatal Tdap compared to White women. ${ }^{12}$ This racial disparity is concerning since Black women are also at higher risk of preterm birth, and preterm babies are most vulnerable to pertussis.

We also observed that women who delivered preterm were less likely to receive prenatal Tdap. This finding is consistent with previous research ${ }^{11}$ and provides justification for earlier Tdap immunization during pregnancy based on recent evidence that earlier Tdap immunization during pregnancy leads to higher neonatal anti-pertussis antibodies and 
encourages immunization to women who subsequently deliver preterm. ${ }^{15,29}$ Future research is needed to address the safety and effectiveness of prenatal Tdap by gestational age at administration.

Further investigation is warranted to understand why pregnant women with PPO or EPO insurance plans and those seeing out-of-network providers were less likely to receive prenatal Tdap. The Affordable Care Act has mandated removal of co-pays associated with vaccines or their administration if vaccines are provided by an in-network provider, therefore, women receiving prenatal care from an out-of-network provider may still need to share the cost of receiving Tdap. Further, an increasing number of providers may no longer be offering vaccines in their offices, due to a reported lack of adequate reimbursement.

Our study had several limitations. First, our study population included only commerciallyinsured women. Given lower uptake of prenatal Tdap among women with Medicaid as compared to privately-insured women, ${ }^{10}$ our findings may not be generalizable to women of lower socioeconomic status. Second, our ascertainment of Tdap immunization may have slightly underestimated vaccine receipt by failing to capture immunizations among women who received prenatal Tdap without requesting insurance reimbursement (e.g., self-payment or community-based free immunization campaigns). Although immunization administration has not been validated in the MarketScan data, our assessment of immunization administration is likely to be well-captured given that insurance claims are the basis for reimbursement, audited for accuracy, and not subject to recall bias, as in commonly used phone surveys or in-person interviews to assess vaccine coverage. Third, information on several potentially important factors (e.g., race, ethnicity, and socioeconomic status) were not available for analysis since the MarketScan databases do not include these variables. We were also unable to reliably identify differences in Tdap receipt by provider type (i.e. obstetrician-gynecologist, family physician, midwife) due to difficulties in assessing the provider of prenatal care services.

Years after the initial ACIP recommendation for prenatal Tdap immunization, less than half of pregnant women in the US are receiving prenatal Tdap immunization. Our findings underscore the urgent need for implementation and dissemination strategies to increase Tdap coverage among pregnant women. Further research is warranted on the effectiveness of interventions to increase demand for Tdap among pregnant women, through strategies such as patient reminder and recall systems, clinic-based approaches, and addressing concerns about vaccine safety.

\section{Supplementary Material}

Refer to Web version on PubMed Central for supplementary material.

\section{Acknowledgments}

Funded by the US National Institute of Allergy and Infectious Diseases (5R21AI115205). The database infrastructure used for this project was funded by the Department of Epidemiology, UNC Gillings School of Global Public Health; the Cecil G. Sheps Center for Health Services Research, UNC; the CER Strategic Initiative of UNC's Clinical \& Translational Science Award (UL1TR001111); and the UNC School of Medicine. 


\section{REFERENCES}

1. Centers for Disease Control and Prevention. 2014 Provisional Pertussis Surveillance Report. 2015

2. Cortese MM, Baughman AL, Zhang R, Srivastava PU, Wallace GS. Pertussis hospitalizations among infants in the United States, 1993 to 2004. Pediatrics. 2008; 121(3):484-492. [PubMed: 18310196]

3. Kline JM, Lewis WD, Smith EA, Tracy LR, Moerschel SK. Pertussis: a reemerging infection. American family physician. 2013; 88(8):507-514. [PubMed: 24364571]

4. Pertussis vaccination: use of acellular pertussis vaccines among infants and young children. Recommendations of the Advisory Committee on Immunization Practices (ACIP). MMWR. Recommendations and reports : Morbidity and mortality weekly report. Recommendations and reports / Centers for Disease Control. 1997; 46(Rr-7):1-25.

5. Auerbach BS, Lake AM, Wilson ME, et al. Dose-response to a two-component acellular pertussis vaccine in infants and comparison with whole-cell vaccine. Biologicals : journal of the International Association of Biological Standardization. 1998; 26(2):145-153. [PubMed: 9811522]

6. Bisgard KM, Rhodes P, Connelly BL, et al. Pertussis vaccine effectiveness among children 6 to 59 months of age in the United States, 1998-2001. Pediatrics. 2005; 116(2):e285-294. [PubMed: 16061582]

7. Updated recommendations for use of tetanus toxoid, reduced diphtheria toxoid and acellular pertussis vaccine (Tdap) in pregnant women and persons who have or anticipate having close contact with an infant aged <12 months --- Advisory Committee on Immunization Practices (ACIP), 2011. MMWR. Morbidity and mortality weekly report. 2011; 60(41):1424-1426. [PubMed: 22012116]

8. Updated recommendations for use of tetanus toxoid, reduced diphtheria toxoid, and acellular pertussis vaccine (Tdap) in pregnant women--Advisory Committee on Immunization Practices (ACIP), 2012. MMWR. Morbidity and mortality weekly report. 2013; 62(7):131-135. [PubMed: 23425962]

9. Murphy TV, Slade BA, Broder KR, et al. Prevention of pertussis, tetanus, and diphtheria among pregnant and postpartum women and their infants recommendations of the Advisory Committee on Immunization Practices (ACIP). MMWR. Recommendations and reports : Morbidity and mortality weekly report. Recommendations and reports / Centers for Disease Control. 2008; 57(Rr-4):1-51.

10. Koepke R, Kahn D, Petit AB, et al. Pertussis and Influenza Vaccination Among Insured Pregnant Women - Wisconsin, 2013-2014. MMWR. Morbidity and mortality weekly report. 2015; 64(27): 746-750. [PubMed: 26182193]

11. Kharbanda EO, Vazquez-Benitez G, Lipkind H, et al. Receipt of pertussis vaccine during pregnancy across 7 Vaccine Safety Datalink sites. Preventive medicine. 2014; 67:316-319. [PubMed: 24952094]

12. Housey M, Zhang F, Miller C, et al. Vaccination with tetanus, diphtheria, and acellular pertussis vaccine of pregnant women enrolled in Medicaid--Michigan, 2011-2013. MMWR. Morbidity and mortality weekly report. 2014; 63(38):839-842. [PubMed: 25254561]

13. Kharbanda EO, Vazquez-Benitez G, Lipkind HS, et al. Maternal Tdap vaccination: Coverage and acute safety outcomes in the vaccine safety datalink, 2007-2013. Vaccine. 2016; 34(7):968-973. [PubMed: 26765288]

14. Abu Raya B, Srugo I, Kessel A, et al. The effect of timing of maternal tetanus, diphtheria, and acellular pertussis (Tdap) immunization during pregnancy on newborn pertussis antibody levels - a prospective study. Vaccine. 2014; 32(44):5787-5793. [PubMed: 25173476]

15. Eberhardt CS, Blanchard-Rohner G, Lemaitre B, et al. Maternal Immunization Earlier in Pregnancy Maximizes Antibody Transfer and Expected Infant Seropositivity Against Pertussis. Clinical infectious diseases : an official publication of the Infectious Diseases Society of America. 2016; 62(7):829-836. [PubMed: 26797213]

16. Vilajeliu A, Gonce A, Lopez M, et al. Combined tetanus-diphtheria and pertussis vaccine during pregnancy: transfer of maternal pertussis antibodies to the newborn. Vaccine. 2015; 33(8):10561062. [PubMed: 25573035] 
17. Healy CM, Rench MA, Baker CJ. Importance of timing of maternal combined tetanus, diphtheria, and acellular pertussis (Tdap) immunization and protection of young infants. Clinical infectious diseases : an official publication of the Infectious Diseases Society of America. 2013; 56(4):539544. [PubMed: 23097585]

18. Martin, JA., Ventura, SJ., et al. National vital statistical reports. Vol. 61. National Center for Health Statistics; Hyattsville, MD: 2012. Births: Final data for 2010. HB

19. Markus AR, Andres E, West KD, Garro N, Pellegrini C. Medicaid covered births, 2008 through 2010 , in the context of the implementation of health reform. Women's health issues : official publication of the Jacobs Institute of Women's Health. 2013; 23(5):e273-280.

20. Centers for Disease Control and Prevention. VFC childhood vaccine supply policy 2009. http:// www.cdc.gov/vaccines/programs/vfc/about/vac-supply-policy/supply-2009.html. Accessed July 8, 2016

21. Castagnini LA, Healy CM, Rench MA, Wootton SH, Munoz FM, Baker CJ. Impact of maternal postpartum tetanus and diphtheria toxoids and acellular pertussis immunization on infant pertussis infection. Clinical infectious diseases : an official publication of the Infectious Diseases Society of America. 2012; 54(1):78-84. [PubMed: 22075790]

22. Healy CM, Rench MA, Wootton SH, Castagnini LA. Evaluation of the impact of a pertussis cocooning program on infant pertussis infection. The Pediatric infectious disease journal. 2015; 34(1):22-26. [PubMed: 24992123]

23. The American College of Obstetricians and Gynecologists: Immunization Expert Work Group. Washington: Immunization Coding for Obstetrician-Gynecologists 2016. DC2016

24. United States Census Bureau. Metropolitan and micropolitan statistical areas main. http:// www.census.gov/population/metro/. Accessed June 24, 2016

25. Notice to readers: final 2012 reports of nationally notifiable infectious diseases. MMWR. Morbidity and mortality weekly report. 2013; 62(33):669-682. [PubMed: 24133698]

26. Ding H, Black CL, Ball S, et al. Influenza Vaccination Coverage Among Pregnant Women--United States, 2014-15 Influenza Season. MMWR. Morbidity and mortality weekly report. 2015; 64(36): 1000-1005. [PubMed: 26390253]

27. Wong CY, Thomas NJ, Clarke M, Boros C, Tuckerman J, Marshall HS. Maternal uptake of pertussis cocooning strategy and other pregnancy related recommended immunizations. Human vaccines \& immunotherapeutics. 2015; 11(5):1165-1172. [PubMed: 25874807]

28. van Lier A, Steens A, Ferreira JA, van der Maas NA, de Melker HE. Acceptance of vaccination during pregnancy: experience with 2009 influenza A (H1N1) in the Netherlands. Vaccine. 2012; 30(18):2892-2899. [PubMed: 22374374]

29. Kent A, Ladhani SN, Andrews NJ, et al. Pertussis Antibody Concentrations in Infants Born Prematurely to Mothers Vaccinated in Pregnancy. Pediatrics. 2016

30. O'Halloran AC, Lu PJ, Williams WW, Ding H, Meyer SA. Tetanus, diphtheria, and acellular pertussis vaccination among women of childbearing age-United States, 2013. American journal of infection control. 2016; 44(7):786-793. [PubMed: 27372388] 


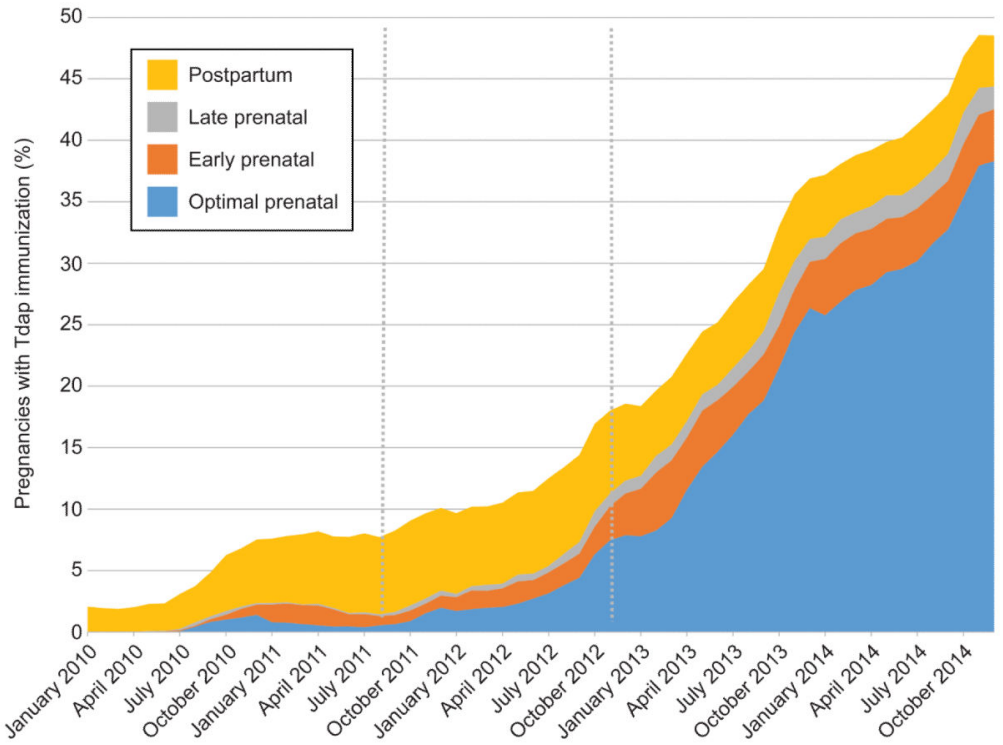

Figure 1.

Percent of pregnancies with tetanus toxoid, reduced diphtheria toxoid, and acellular pertussis (Tdap) immunization, by delivery month and timing of vaccine administration, 2010-2014. Grey dotted lines denote the June 2011 Advisory Committee on Immunization Practices (ACIP) recommendations for Tdap for unvaccinated pregnant women and October 2012 ACIP recommendations for Tdap immunization with each pregnancy. Results did not change appreciably in the sensitivity analyses that excluded 1,695 (0.1\%) women who delivered before 27 weeks of gestation. 


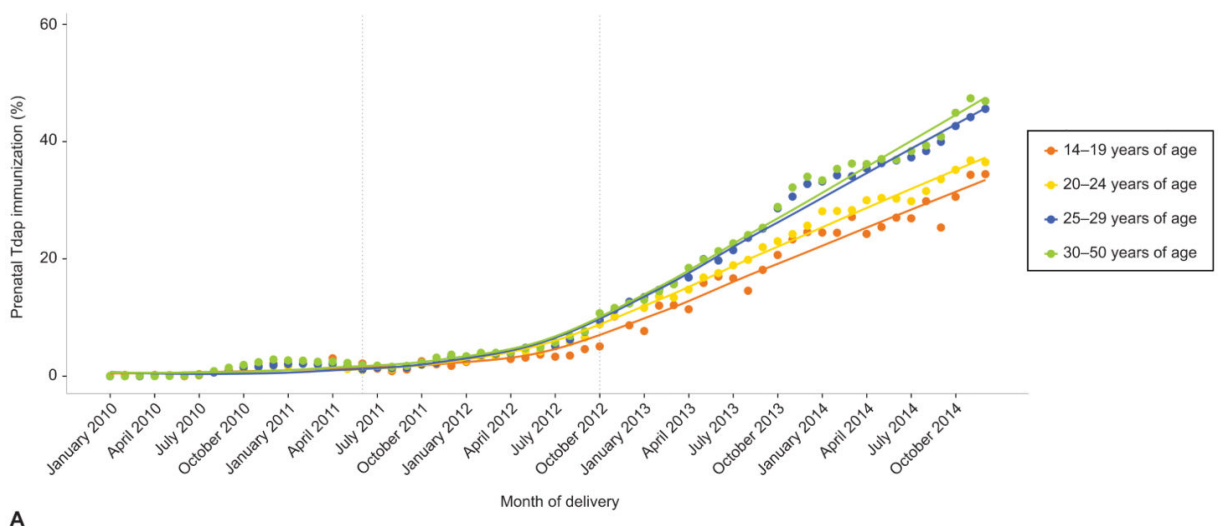

A

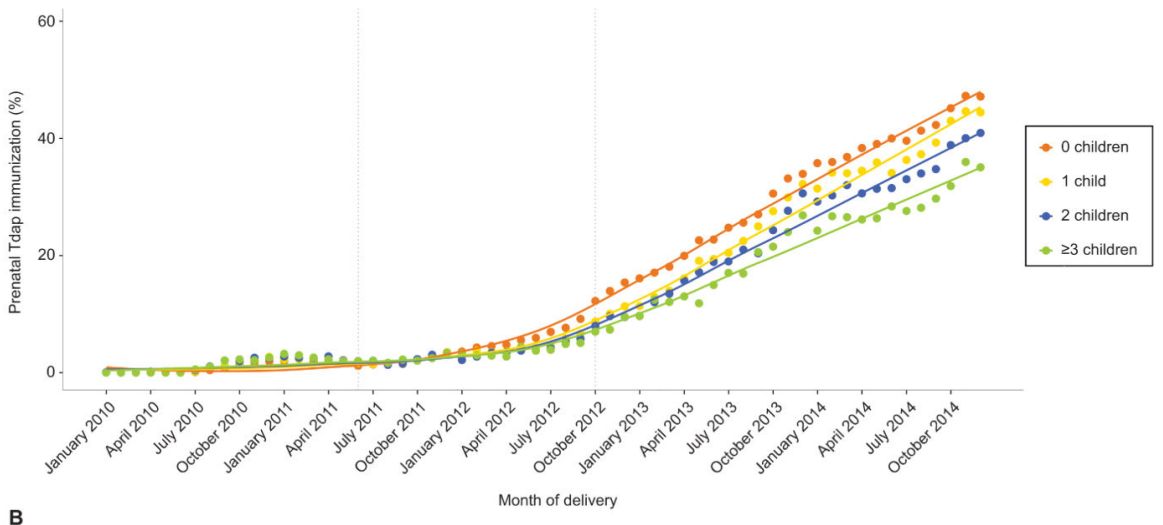

B

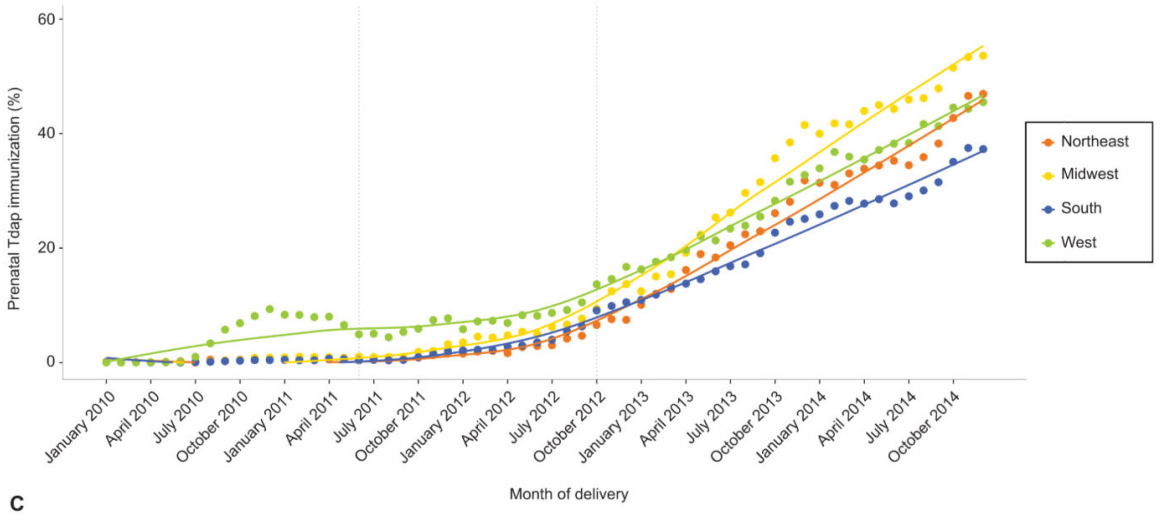

Figure 2.

Proportion of pregnancies with prenatal tetanus toxoid, reduced diphtheria toxoid, and acellular pertussis (Tdap) immunization receipt by delivery month, 2010-2014, by maternal age (A), number of other dependent children (B), geographic region of residence $(\mathbf{C})$. Grey dotted lines denote the June 2011 Advisory Committee on Immunization Practices (ACIP) recommendations for Tdap for unvaccinated pregnant women and October 2012 ACIP recommendations for Tdap immunization with each pregnancy. 


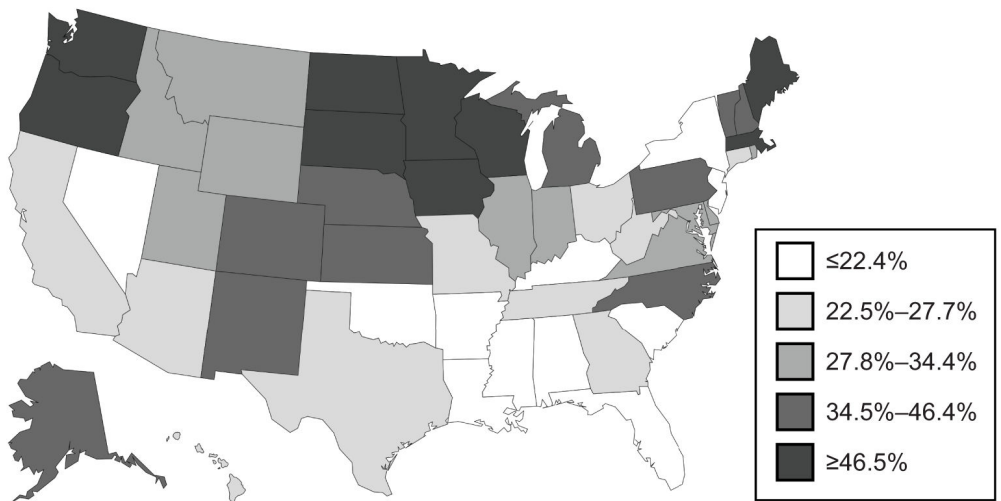

Figure 3.

Geographic distribution of prenatal tetanus toxoid, reduced diphtheria toxoid, and acellular pertussis (Tdap) immunization (\%) by state, November 2012-December 2014. Analysis was restricted to the time period following the October 2012 Advisory Committee on Immunization Practices (ACIP) recommendation. 


\begin{tabular}{ll}
\hline Variable & aRR (95\% CI*) \\
\hline Maternal age (years) & $0.83(0.80-0.85)$ \\
$14-19$ vs. 30-34 & $0.87(0.85-0.88)$ \\
$20-24$ vs. 30-34 & $0.95(0.94-0.96)$ \\
$25-29$ vs. 30-34 & $1.01(0.99-1.03)$ \\
$35-39$ vs. 30-34 & $0.97(0.94-1.01)$ \\
$40-50$ vs. 30-34 & $0.98(0.97-0.99)$ \\
Number of siblings & $0.93(0.92-0.95)$ \\
1 vs. 0 & $0.86(0.84-0.88)$ \\
2 vs. 0 & $0.99(0.92-1.06)$ \\
3 or more vs. 0 & $0.93(0.92-0.95)$ \\
Overnight hospitalization & $0.93(0.92-0.95)$ \\
Yes vs. no & $0.62(0.58-0.66)$ \\
Emergency department visit & $1.06(1.04-1.08)$ \\
Yes vs. no & $1.03(1.01-1.05)$ \\
Network of provider type & $1.11(1.09-1.13)$ \\
Out-of-network or mix-of-networks vs. in-network & $1.10(1.08-1.12)$ \\
No service vs. in-network & \\
Health plan type & $0.84(0.83-0.86)$ \\
HMO vs. PPO/EPO & $0.81(0.80-0.82)$ \\
POS/POS with capitation vs. PPO/EPO & $0.82(0.81-0.83)$ \\
CDHP vs. PPO/EPO & $1.03(1.02-1.05)$ \\
Other & \\
Region of residence & \\
Northeast vs. Midwest & \\
South vs. Midwest & \\
West vs. Midwest & \\
Metropolitan statistical area & \\
Yes vs. no & \\
\hline &
\end{tabular}

Figure 4.

Adjusted rate ratio estimates for the associations between prenatal tetanus toxoid, reduced diphtheria toxoid, and acellular pertussis (Tdap) vaccine receipt and patient characteristics among pregnant women, November 2012-December 2014. A total of 14,829 (6.4\%) of mothers were excluded from these analyses due to missing data on at least one potential predictor (ie, health insurance type, residence within a metropolitan statistical area, and/or region of residence). aRR, adjusted rate ratio; CI, confidence interval; HMO, Health Maintenance Organization; PPO, Preferred Provider Organization; EPO, Exclusive Provider Organization; POS, Point of Service; CDHP, Consumer Directed Health Plan. 


\section{Table 1}

Descriptive characteristics of pregnancies by timing of Tdap immunization, 2010-2014.*

\begin{tabular}{|c|c|c|c|c|}
\hline \multirow[b]{2}{*}{ Characteristic } & \multicolumn{4}{|c|}{$\mathbf{N}(\%)$} \\
\hline & $\begin{array}{c}\text { Prenatal } \\
\mathrm{n}=175,844 \\
(14.4 \%)\end{array}$ & $\begin{array}{c}\text { Postpartum } \\
\mathbf{n}=65,699 \\
(5.4 \%)\end{array}$ & $\begin{array}{c}\text { None } \\
\mathbf{n}=\mathbf{9 8 0 , 8 4 1} \\
(\mathbf{8 0 . 2 \% )}\end{array}$ & $\begin{array}{c}\text { Total } \\
\mathbf{n}=\mathbf{1 , 2 2 2 , 3 8 4} \\
(\mathbf{1 0 0 . 0 \%})\end{array}$ \\
\hline \multicolumn{5}{|l|}{ Year of delivery } \\
\hline 2010 & $1,690(1.0)$ & $6,079(9.3)$ & $179,842(18.3)$ & $187,611(15.4)$ \\
\hline 2011 & $5,519(3.1)$ & $15,841(24.1)$ & $232,822(23.7)$ & $254,182(20.8)$ \\
\hline 2012 & $19,019(10.8)$ & $19,575(29.8)$ & $251,585(25.7)$ & $290,179(23.7)$ \\
\hline 2013 & $49,901(28.4)$ & $11,999(18.3)$ & $164,212(16.7)$ & $226,112(18.5)$ \\
\hline 2014 & $99,715(56.7)$ & 12,205 (18.6) & $152,380(15.5)$ & $264,300(21.6)$ \\
\hline $\begin{array}{l}\text { Maternal age at pregnancy } \\
\text { onset (years), median (SD) }\end{array}$ & $30(5.1)$ & $29.0(5.3)$ & $30.0(5.4)$ & $30.0(5.3)$ \\
\hline \multicolumn{5}{|l|}{$\begin{array}{l}\text { Number of other dependent } \\
\text { children }\end{array}$} \\
\hline 0 & $76,946(43.8)$ & $27,862(42.4)$ & $366,817(37.4)$ & 471,625 (38.6) \\
\hline 1 & $58,780(33.4)$ & $20,871(31.8)$ & $331,085(33.8)$ & $410,736(33.6)$ \\
\hline 2 & $27,412(15.6)$ & $11,028(16.8)$ & $181,680(18.5)$ & $220,120(18.0)$ \\
\hline$\geq 3$ & $12,706(7.2)$ & $5,938(9.0)$ & $101,259(10.3)$ & $119,903(9.8)$ \\
\hline $\begin{array}{l}\text { Obstetric blood panel prior to } 14 \\
\text { weeks gestation }\end{array}$ & $92,068(52.4)$ & $36,619(55.7)$ & $481,973(49.1)$ & $610,660(50.0)$ \\
\hline $\begin{array}{l}\text { Overnight hospitalization during } \\
\text { pregnancy }\end{array}$ & $1,030(0.6)$ & $552(0.8)$ & $8,304(0.9)$ & $9,886(0.8)$ \\
\hline $\begin{array}{l}\text { Emergency department visit } \\
\text { during pregnancy }\end{array}$ & $22,784(13.0)$ & $9,433(14.4)$ & $136,422(13.9)$ & $168,639(13.8)$ \\
\hline \multicolumn{5}{|l|}{ Network of provider type } \\
\hline In-network & $152,483(86.7)$ & $53,587(81.6)$ & $759,944(77.5)$ & $966,014(79.0)$ \\
\hline $\begin{array}{l}\text { Out of network or mix of } \\
\text { networks }\end{array}$ & $22,207(12.6)$ & $9,567(14.6)$ & $136,264(13.9)$ & $168,038(13.8)$ \\
\hline No service & $1,154(0.7)$ & $2,545(3.9)$ & $84,633(8.6)$ & $88,332(7.2)$ \\
\hline \multicolumn{5}{|l|}{ Health insurance type } \\
\hline PPO or EPO & $108,659(61.8)$ & $45,194(68.8)$ & $645,419(65.8)$ & $799,272(65.4)$ \\
\hline HMO & $23,963(13.6)$ & $7,242(11.0)$ & $121,744(12.4)$ & $152,949(12.5)$ \\
\hline POS or POS with capitation & $9,802(5.6)$ & $3,149(4.8)$ & $53,768(5.5)$ & $66,719(5.5)$ \\
\hline $\mathrm{CDHP}$ & $13,555(7.7)$ & $3,585(5.5)$ & $61,842(6.3)$ & $78,982(6.5)$ \\
\hline Other & $14,554(8.3)$ & $3,825(5.8)$ & $56,339(5.7)$ & $74,718(6.1)$ \\
\hline Missing & $5,311(3.0)$ & $2,704(4.1)$ & $41,729(4.3)$ & $49,744(4.1)$ \\
\hline \multicolumn{5}{|l|}{ Region of residence } \\
\hline Northeast & $27,189(15.5)$ & $8,936(13.6)$ & $156,092(15.9)$ & $192,217(15.7)$ \\
\hline North Central & $46,936(26.7)$ & $14,442(22.0)$ & $233,437(23.8)$ & $294,815(24.1)$ \\
\hline South & $48,669(27.7)$ & $26,029(39.6)$ & $364,896(37.2)$ & $439,594(36.0)$ \\
\hline West & $47,493(27.0)$ & $14,566(22.2)$ & $201,844(20.6)$ & $263,903(21.6)$ \\
\hline Unknown & $5,557(3.2)$ & $1,726(2.6)$ & $24,572(2.5)$ & $31,855(2.6)$ \\
\hline
\end{tabular}




\begin{tabular}{lllll}
\hline \multicolumn{5}{c}{$\mathbf{N}(\%)$} \\
\hline Characteristic & $\begin{array}{c}\text { Prenatal } \\
\mathbf{n = 1 7 5 , 8 4 4} \\
\mathbf{( 1 4 . 4 \% )}\end{array}$ & $\begin{array}{c}\text { Postpartum } \\
\mathbf{n}=\mathbf{6 5 , 6 9 9} \\
\mathbf{( 5 . 4 \% )}\end{array}$ & $\begin{array}{c}\text { None } \\
\mathbf{n = 9 8 0 , 8 4 1} \\
\mathbf{( 8 0 . 2 \% )}\end{array}$ & $\begin{array}{c}\text { Total } \\
\mathbf{n}=\mathbf{1 , 2 2 2 , 3 8 4} \\
\mathbf{( 1 0 0 . 0 \% )}\end{array}$ \\
\hline No & $17,150(9.8)$ & $7,935(12.1)$ & $124,623(12.7)$ & $149,708(12.3)$ \\
Yes & $153,138(87.1)$ & $56,039(85.3)$ & $831,692(84.8)$ & $1,040,869(85.2)$ \\
Missing & $5,556(3.2)$ & $1,725(2.6)$ & $24,526(2.5)$ & $31,807(2.6)$ \\
\hline
\end{tabular}

Abbreviations: CDHP, Consumer Directed Health Plan; EPO, Exclusive Provider Organization; HMO, Health Maintenance Organization; POS, Point of Service; PPO, Preferred Provider Organization; SD, standard deviation. 